\title{
REGISTRO DE ARTROPLASTIAS DO OMBRO
}

\author{
SHOULDER ARTHROPLASTY RECORDS
}

Geraldo Motta Filho', Marcus Vinicius Galvão², Martim Monteiro², Marcio Cohen², Bruno Brandão²

\section{RESUMO}

Objetivo: Determinar as características clínico-cirúrgicas referentes a 145 artroplastias do ombro realizadas no período entre julho de 2004 a dezembro de 2006. Métodos: No período de estudo foram realizadas 145 artroplastias de ombro. Através de protocolo prospectivo, informações demográficas, da anamnese, exame físico e exame radiográfico foram armazenadas em um banco de dados. Os dados coletados foram organizados em três grandes grupos: doenças articulares degenerativas, fraturas e seqüelas traumáticas. Esses dados foram correlacionados a fim de definir o perfil epidemiológico dos pacientes, das lesões e artroplastias. Resultados: Das 145 artroplastias de ombro realizadas $37 \%$ foram por seqüelas traumáticas, 30\% por doença articular degenerativa e 33\% com diagnóstico de fratura. Foram 12\% artroplastias totais e $88 \%$ parciais. Ocorreram cinco complicações no pós-operatório recente. Conclusão: As artroplastias de ombro tornaram-se um procedimento frequente na prática ortopédica. Registros cirúrgicos são importantes a fim de demonstrar essa evolução progressiva e permitir avaliações de resultados clínicos no futuro.

Descritores - Ombro; Artroplastia; Registros médicos; Estudos prospectivos

\section{ABSTRACT}

Objective: The study's objective is to evaluate the characteristics and problems of patients who underwent shoulder arthroplasties between July 2004 and November 2006. Methodology: During the period of the study, 145 shoulder arthroplasties were performed. A prospective protocol was used for every patient; demographic, clinical and surgical procedure data were collected. All gathered data were included in the data base. The patients were divided in three major groups: fractures, degenerative diseases and trauma sequels. Information obtained from the data base was correlated in order to determine patients' epidemiologic, injuries, and surgical procedure profiles. Results: Of the 145 shoulder arthroplasties performed, $37 \%$ presented trauma sequels, 30\% degenerative diseases, and 33\% proximal humerus fracture. $12 \%$ of the cases required total arthroplasties and 88\% partial arthroplasties. Five major complications were observed on early postoperative period. Conclusion: Shoulder arthroplasties have become a common procedure in orthopaedic practice. Surgical records are important in evidencing progressive evolution and in enabling future clinical outcomes evaluation.

Keywords - Shoulder; Arthroplasty; Medical records; Prospective studies

\section{INTRODUÇÃO}

Segundo Lugli, foi o cirurgião francês Péan que, em 1893, realizou a primeira substituição da articulação do ombro ${ }^{(1)}$. Nos anos 1950, a partir de relatos de Neer, as artroplastias de ombro evoluíram com aumento das indicações e melhores resultados clínicos, em virtude de maior entendimento biomecânico do ombro, evolução do desenho das próteses e da técnica cirúrgica ${ }^{(2)}$.

A melhoria dos resultados clínicos das artroplastias, associada ao envelhecimento da população e à valorização da qualidade de vida, tornou a artroplastia de ombro um procedimento mais frequente na prática ortopédica $^{(3)}$.

Em diversas séries foi demonstrado que a substituição artroplástica do ombro tem produzido resultados satisfatórios. Infelizmente, os resultados da maioria das

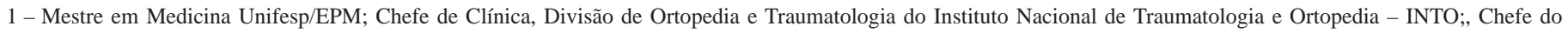
Serviço de Cirurgia do Ombro e Cotovelo do Instituto Nacional de Traumatologia e Ortopedia - INTO.

2 - Médico do Serviço de Cirurgia do Ombro e Cotovelo do Instituto Nacional de Traumatologia e Ortopedia - INTO.
}

Trabalho realizado no Serviço de Cirurgia do Ombro e Cotovelo do Instituto Nacional de Traumatologia e Ortopedia - INTO/RJ, Brasil.

Correspondência: Marcus Vinícius Galvão - Av. Afrânio de Melo Franco, 141, sala 208 - 22430-060 - Rio de Janeiro, RJ. E-mail: mvgamaral@terra.com.br 
artroplastias realizadas não estão disponíveis. Isso ocorre em grande parte devido à maioria dos sistemas de avaliação de resultados necessitarem de grande dedicação dos profissionais envolvidos, além de serem muito onerosos para a maioria dos cirurgiões que realizam substituições articulares do ombro, configurando um desestímulo à realização de estudos relacionados a esse procedimento cirúrgico ${ }^{(4)}$.

Registros cirúrgicos permitem à comunidade médica obter informações em relação à epidemiologia dos procedimentos cirúrgicos, sua técnica, implantes e seus resultados, sendo uma ferramenta útil na evolução dos procedimentos ortopédicos ${ }^{(5)}$.

Dessa forma, o objetivo deste artigo é analisar os dados armazenados no registro de artroplastias do ombro realizadas pelo Serviço de Cirurgia de Ombro e Cotovelo (SCOC), do Instituto Nacional de Traumatologia e Ortopedia (INTO), a fim de documentar as características epidemiológicas dos pacientes submetidos a esse procedimento, definindo o perfil clínico-cirúrgico e as variáveis técnicas relacionadas a esse tipo de cirurgia ortopédica e, futuramente, realizar análises funcionais desses pacientes.

\section{MÉTODOS}

Em agosto de 2004 foi iniciado o registro cirúrgico das artroplastias de ombro realizadas pelo Serviço de Cirurgia de Ombro e Cotovelo (SCOC) do Instituto Nacional de Traumatologia e Ortopedia - INTO.

A partir dessa data, todas as artroplastias de ombro realizadas pelo SCOC no Hospital são documentadas por meio de protocolo específico (Figura 1), preenchido pelo estagiário ou médico do Serviço, no momento da admissão do paciente e complementado após o procedimento. Além de dados pessoais do paciente, no protocolo constam dados referentes à doença, avaliação funcional pré-operatória (todos os pacientes, exceto os com fraturas da extremidade proximal do úmero) e informações referentes a procedimento cirúrgico, como técnica cirúrgica e implante.

Os protocolos preenchidos, semanalmente, são lançados em um banco de dados (Microsoft Excel ${ }^{R}$ ), permitindo análise imediata dos dados referentes aos procedimentos. Dessa forma, analisamos 145 artroplastias de ombro realizadas no período entre agosto de 2004 e novembro de 2006 pelo SCOC do INTO, procurando identificar as informações epidemiológicas relacionadas a esse procedimento.
Foram incluídas nessa análise todas as artroplastias de ombro realizadas nesse período pelo nosso Serviço, sendo somente excluídas as artroplastias de ressecção.

Os pacientes foram distribuídos em três grandes grupos de acordo com o diagnóstico: doenças articulares degenerativas, trauma agudo e sequelas traumáticas. O grupo de trauma agudo incluiu as fraturas complexas da extremidade proximal do úmero com evolução temporal menor que seis semanas. O diagnóstico de sequela traumática foi definido como qualquer lesão traumática (osteonecrose pós-traumática, fratura, fratura-luxação, luxação inveterada, pseudartrose, consolidação viciosa) com evolução temporal igual a ou maior do que seis semanas.

O grupo das fraturas foi dividido em subgrupos de acordo com a etiologia. O grupo do trauma agudo foi dividido em subgrupos segundo a classificação de Neer para fraturas da extremidade proximal do úmero e de acordo com a presença e direção de luxação associa$\mathrm{da}^{(6)}$; as sequelas traumáticas foram divididas em subgrupos de acordo com a classificação de Boileau et al ${ }^{(7)}$ (Figura 2).

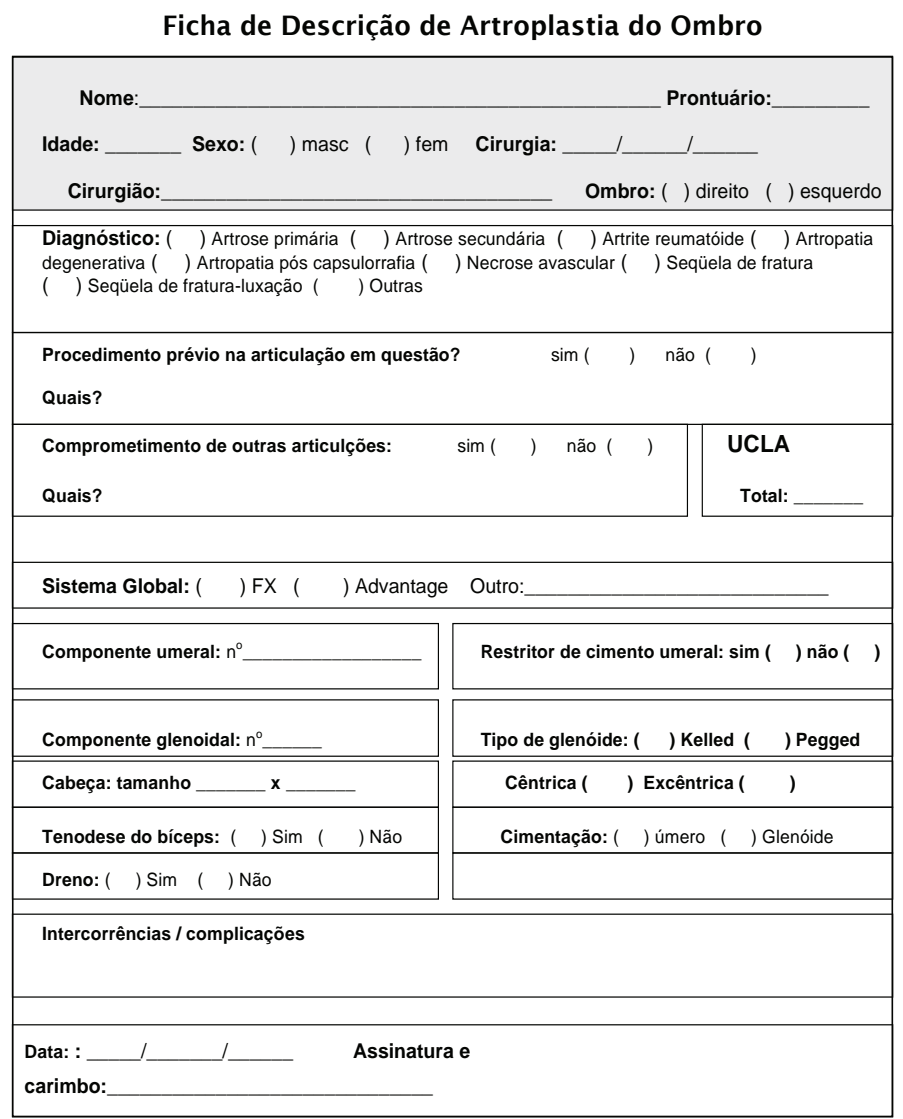

Figura 1 - Protocolo de artroplastia do ombro para aquisição de dados para o registro de artroplastia do ombro e cotovelo do Instituto Nacional de Traumatologia e Ortopedia - INTO 


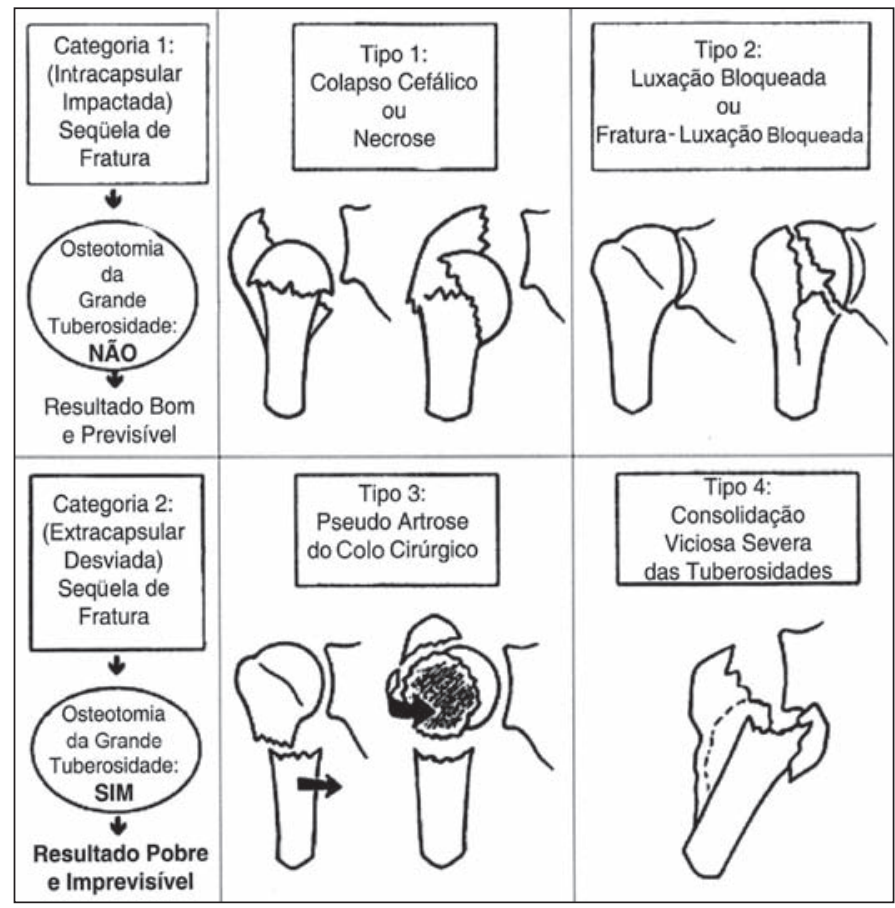

Figura 2 - Classificação de Boileau e Trojani para as sequelas pós-traumáticas da articulação do ombro(7)

Todos os procedimentos cirúrgicos utilizaram próteses do Global Total Shoulder System (De Puy Inc. ${ }^{\circledR}$, Warsaw, IN, EUA.).

Das 145 artroplastias de ombro realizadas, 106 pacientes eram do sexo feminino (73\%) e 39 do masculino (27\%). A média de idade geral foi de 65 anos (30-84 anos). Foram 17 (12\%) artroplastias totais e 128 (88\%) parciais (Gráfico 1). Todas as artroplastias foram realizadas por acesso deltopeitoral. Na divisão por grupos de doenças, 54 (37\%) pacientes apresentavam sequelas traumáticas; 48 (33\%), trauma agudo; e 43 (30\%), diagnóstico de doença articular degenerativa (Tabela 1).

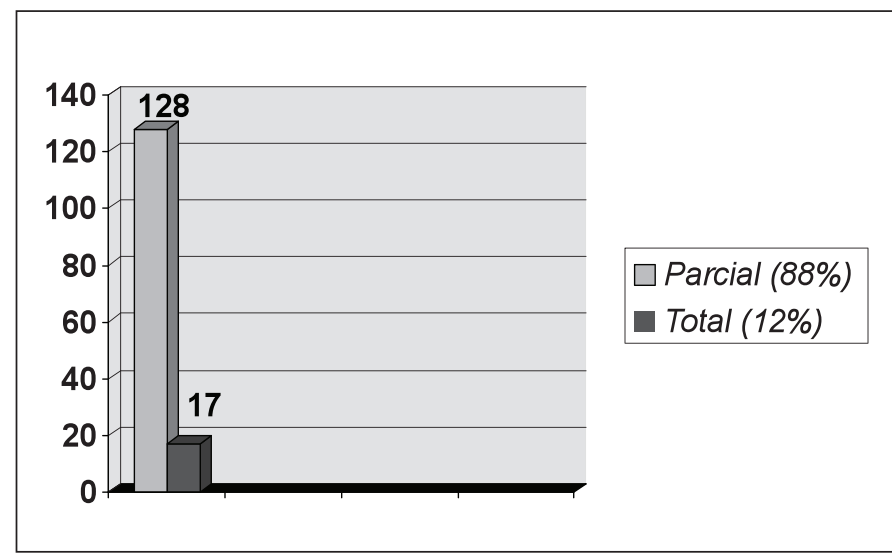

Gráfico 1 - Número de artroplastias parciais e totais no período
Tabela 1 - Registro de Artroplastias do Ombro e Cotovelo

\begin{tabular}{c|c|c}
\hline Registro de Artroplastia do INTO & Número de Pacientes & Total = 145 \\
\hline Doenças Degenerativas & 43 & $30 \%$ \\
\hline Fraturas & 48 & $33 \%$ \\
\hline Seqüelas Traumáticas & 54 & $37 \%$ \\
\hline Fonte: Instituto Nacio
\end{tabular}

\section{RESULTADOS}

No grupo de sequelas traumáticas havia 54 pacientes (37\%). Desses, a média de idade foi de 61 anos (30-81 anos), sendo 40 pacientes do sexo feminino (74\%) e 14 do masculino (26\%). A principal etiologia das sequelas traumáticas de acordo com a classificação de Boileau et $\mathrm{al}^{(7)}$ foi a fratura-luxação inveterada ou luxação inveterada em 22 casos (41\%), seguida pelas consolidações viciosas em 11 casos (20\%), assim como as pseudartroses da extremidade proximal do úmero, também em 11 casos (20\%) e, por fim, as necroses em 10 casos (19\%) (Gráfico 2). O período médio entre a lesão e a cirurgia foi de 23 meses (2-180 meses). O escore funcional da UCLA pré-operatório desse grupo foi de oito pontos. Nesse grupo, foram 44 artroplastias parciais (81\%) e 10 totais (19\%) (Gráfico 3). Todos os componentes glenoideos foram cimentados. Os componentes umerais foram cimentados em 41 pacientes (76\%) e não cimentados em 13 (24\%). Dois pacientes com diagnóstico de pseudartrose da extremidade proximal do úmero com grande perda óssea, metáfiso-diafisária, foram submetidos à enxertia óssea homóloga, sendo um com enxerto estrutural tipo régua cortical e outro com enxerto esponjoso, ambos de doador cadáver, captados pelo Banco de Tecidos MúsculoEsqueléticos do INTO. Cabeça umeral excêntrica foi utilizada em oito casos (15\%).

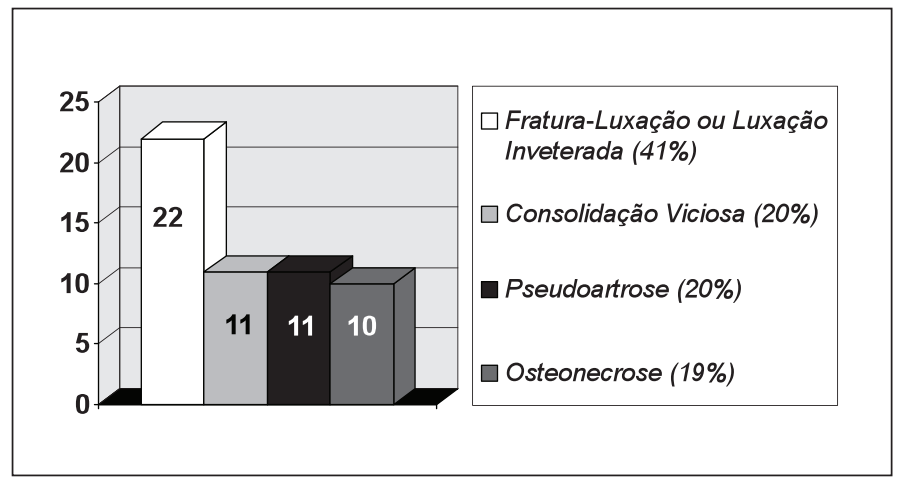

Gráfico 2 - Percentuais de pacientes em cada grupo de acordo com as características do trauma inicial 


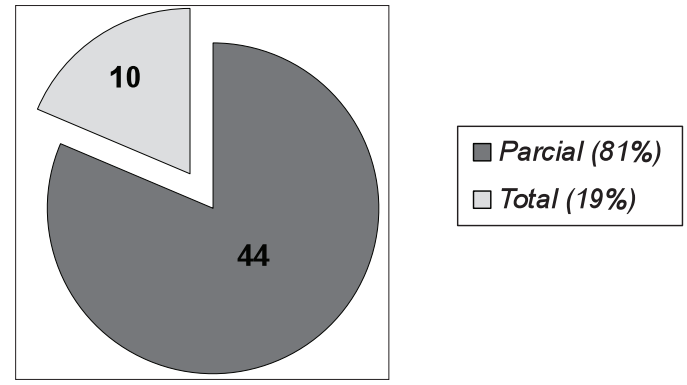

Gráfico 3 - No grupo das sequelas pós-traumáticas foram realizadas $81 \%$ de artroplastias parciais e $19 \%$ totais
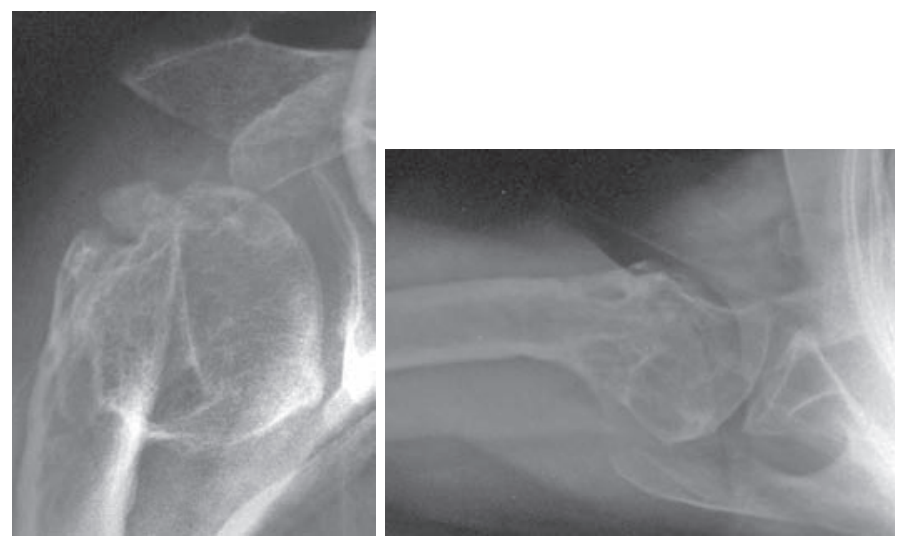

Figura 3 - Imagens radiográficas em AP e perfil ilustrando sequela pós-traumática com as tuberosidades e cabeça umeral consolidadas viciosamente
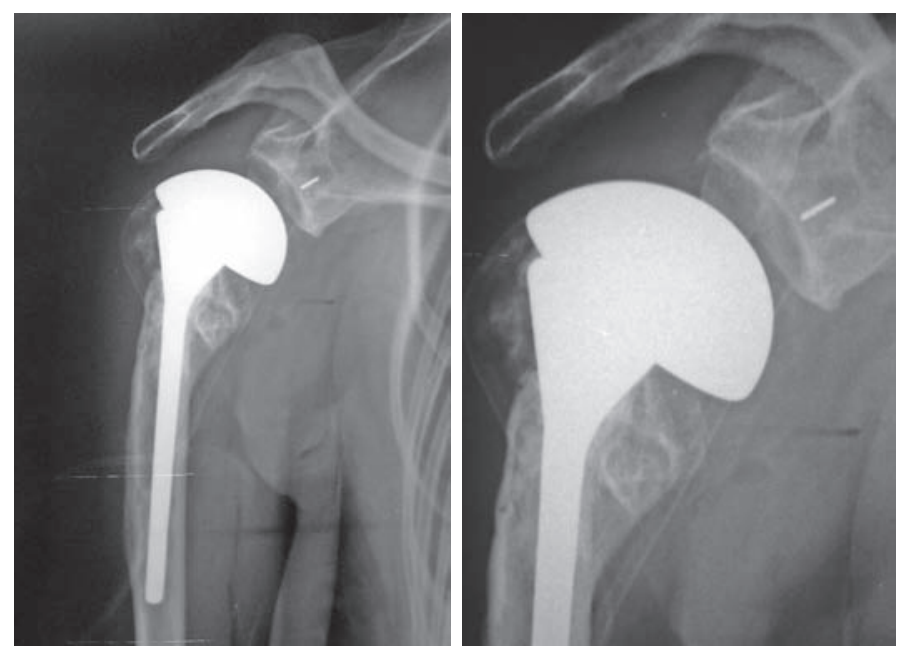

Figura 4 - Imagens radiográficas em AP e perfil ilustrando artroplastia total realizada sem osteotomia dos tubérculos maior e menor

No grupo das doenças degenerativas havia 43 pacientes (30\%) com idade média de 64 anos (26-88 anos), sendo 30 do sexo feminino (70\%) e 13 do masculino (30\%). O diagnóstico mais comum foi de artrose primária em 29 pacientes (67\%). Outros diagnósticos foram de seis casos de artrite reumatoide (14\%), cinco casos de artropatia do manguito rotador (11\%), dois casos de artrose pós-capsuloplastia e um caso de espondilite anquilosante (Gráfico 4). Desse grupo, 14 (32\%) pacientes haviam sido submetidos a procedimentos artroplásticos prévios em qualquer articulação, sendo seis (14\%) casos no ombro contralateral, cinco pacientes com artroplastia de joelho prévia (12\%), duas com substituição articular do quadril (4\%) e uma paciente com artroplastia do joelho e do ombro (2\%). O escore funcional da UCLA pré-operatório desse grupo de pacientes foi de 10 pontos. Nesse grupo foram realizadas 36 artroplastias parciais (83\%) e sete totais (16\%) (Gráfico 5). Todos os componentes glenoideos foram cimentados. Nesse grupo, três pacientes jovens com diagnóstico de osteoartrose primária, com degeneração da superfície articular da glenoide, foram submetidos a recobrimento biológico da glenoide. Dois pacientes submeteram-se a hemiartroplastia associada à interposição biológica homóloga da superfície articular da glenoide com enxerto de menisco lateral de doador cadáver captado do Banco de Tecidos Músculo-Esqueléticos do INTO, e um paciente foi submetido à interposição biológica com enxerto biológico autólogo de cápsula anterior retirado durante o acesso deltopeitoral. Os componentes umerais foram não cimentados em 31 pacientes (72\%) e cimentados em 12 (28\%). Cabeça excêntrica foi utilizada em 10 casos (23\%).

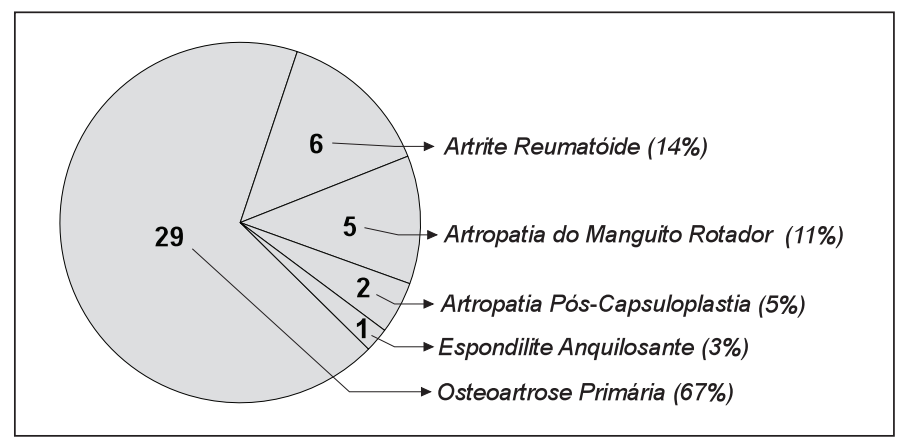

Gráfico 4 - Percentuais de pacientes de acordo com a etiologia no grupo das doenças degenerativas

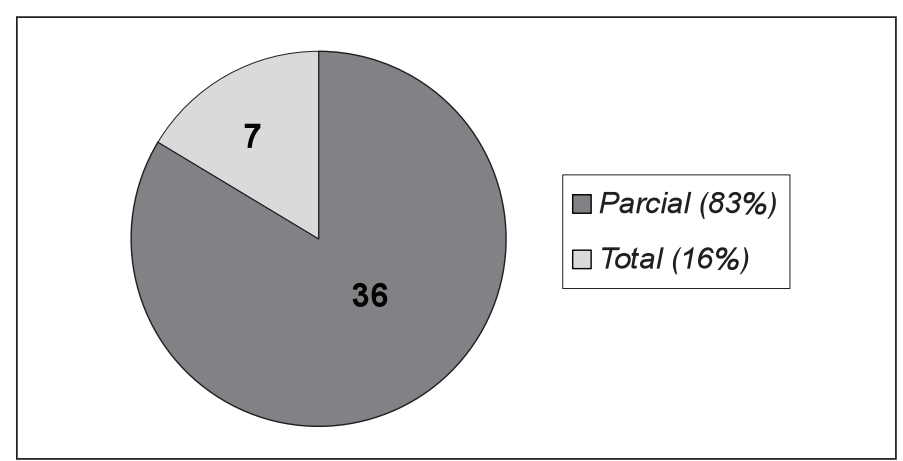

Gráfico 5 - Percentuais de pacientes submetidos a artroplastias parciais e totais no grupo das doenças degenerativas 

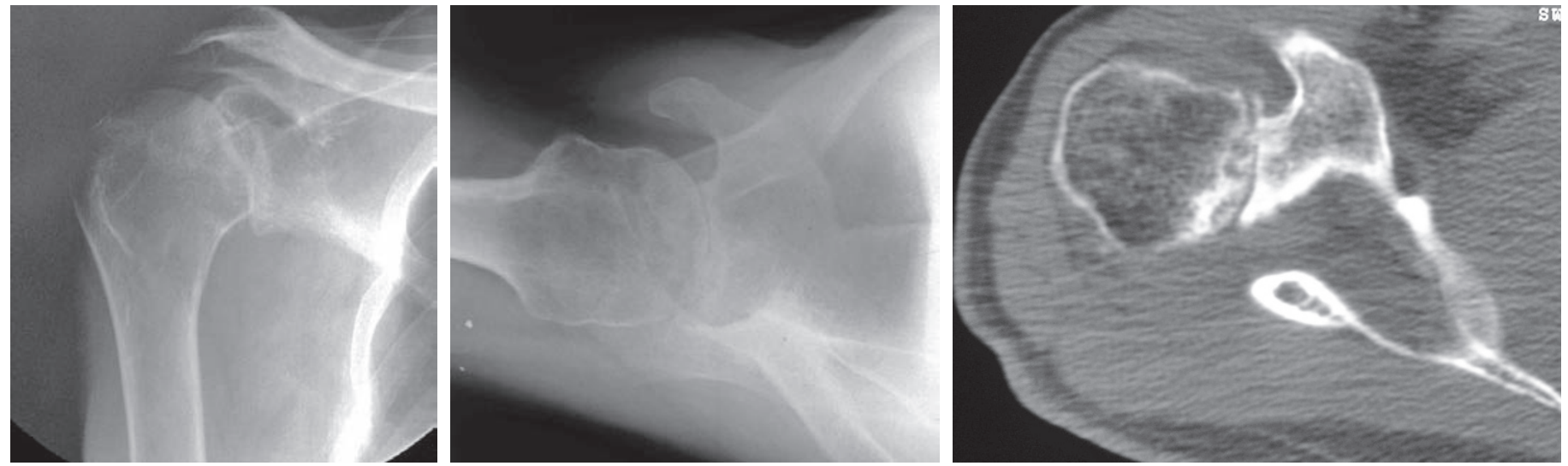

Figura 5 - Imagens radiográficas em AP, perfil e de tomografia computadorizada ilustrando artrose da articulação do ombro
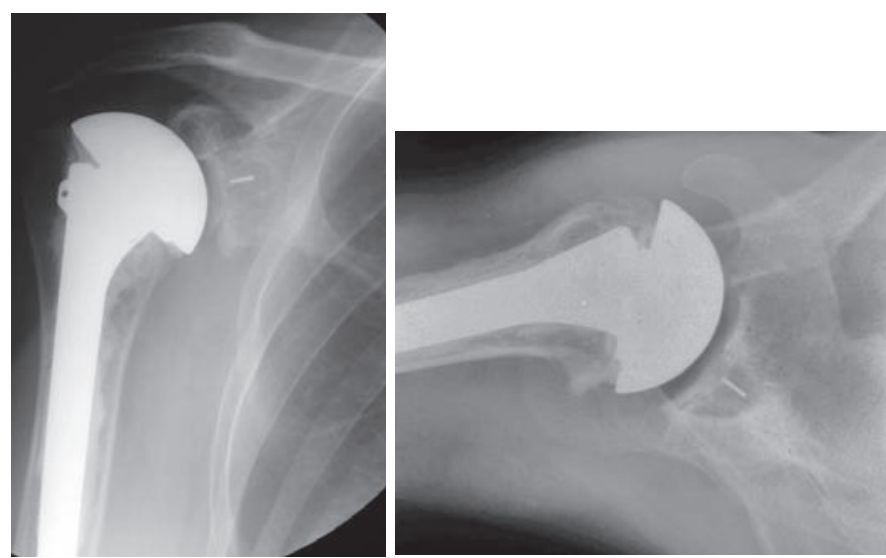

Figura 6 - Imagens radiográficas em AP e perfil ilustrando artroplastia total

Por fim, no grupo dos pacientes com diagnóstico de trauma agudo havia 48 pacientes (33\%) com idade média de 70 anos, sendo 36 do sexo feminino (75\%) e 12 do masculino (25\%). O lado acometido foi o direito em 27 pacientes (56\%) e o esquerdo em 21 (43\%). O intervalo médio até a cirurgia foi de 20 dias. Utilizando a classificação de Neer, 27 pacientes apresentavam fratura em quatro partes (56\%), 18 tinham fratura em três partes (37\%), em dois havia fratura em duas partes do colo anatômico (4\%) e em um paciente (2\%), luxação posterior com quatro semanas de evolução, irredutível de forma incruenta; nesse caso, através de exames de imagem, mensurou-se um defeito na cabeça umeral maior do que 30\% do seu diâmetro, tendo-se optado pela substituição artroplástica em vez de salvamento (Gráfico 6). Nesse grupo, dos 48 pacientes, 19 tinham luxação associada à fratura (39,5\%), sendo 14 anteriores e cinco posteriores. Apresentando lesões associadas havia um paciente com neuropraxia do plexo braquial, com resolução completa espontânea após três meses do trauma inicial, somente tratada com terapia ocupacional, e um paciente com fratura do colo do fêmur ipsilateral tra-

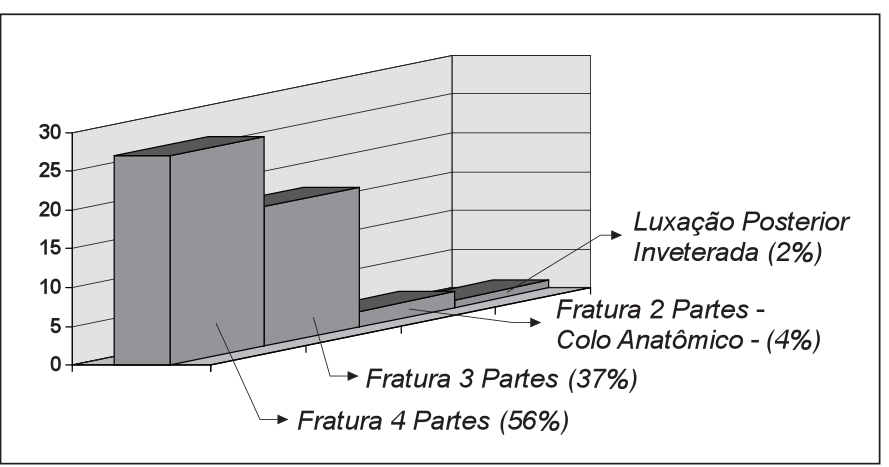

Gráfico 6 - Percentuais de pacientes de acordo com as características do trauma no grupo das lesões traumáticas agudas

tado cirurgicamente com hemiartroplastia em um primeiro tempo cirúrgico pelo serviço de cirurgia de trauma da mesma instituição. Todas as artroplastias foram parciais, sendo 41 (85\%) dos componentes umerais cimentados. Em 14 casos (29\%) foi utilizada cabeça umeral excêntrica.

Como complicações recentes ocorreram: uma lesão da artéria axilar durante o ato operatório em um caso de fratura-luxação anterior inveterada, dois casos de instabilidade, sendo uma anterior e outra posterior, em casos de sequela traumática, uma sepse urinária em um paciente diabético tipo II sem comprometer aparentemente o implante, e o óbito de uma paciente idosa com fratura em decorrência de complicações clinicas pósoperatórias (Gráfico 7).

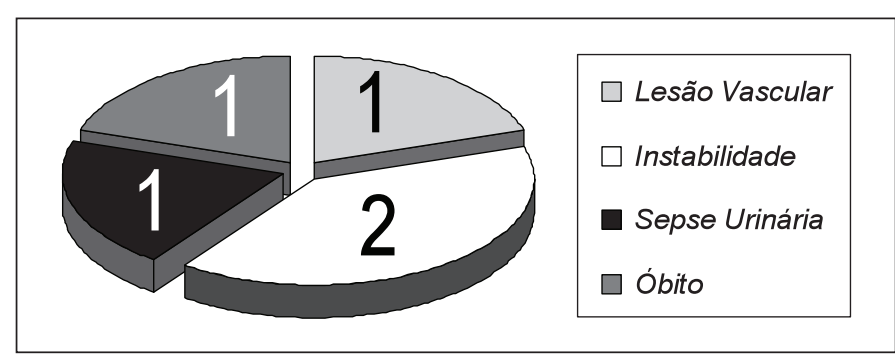

Gráfico 7 - Complicações precoces ocorridas no grupo que foi avaliado 


\section{DISCUSSÃO}

A artroplastia do ombro, ao longo dos anos, temse tornado um procedimento comumente realizado na prática ortopédica ${ }^{(3,8)}$.

Durante todos esses anos, somente 5\% do total das artroplastias do ombro realizadas no mundo tiveram seus resultados publicados, sendo a maioria em grandes centros. Dessa forma, 95\% dos procedimentos foram realizados sem qualquer documentação cientifica. Em virtude disso, a característica epidemiológica e os resultados das artroplastias de ombro são malcaracterizados na literatura especializada, quando comparados com os de outras articulações ${ }^{(4)}$.

Hasan et $\mathrm{l}^{(9)}$ estudaram a distribuição de artroplastias de ombro entre cirurgiões. Observaram que a maioria das artroplastias de ombro nos EUA é realizada por cirurgiões que executam poucos procedimentos semelhantes ao ano. Entretanto a maioria dos resultados na literatura especializada deriva da prática de cirurgiões experientes; assim, é impossível saber os verdadeiros resultados da artroplastia de ombro em seu principal contexto, que é a prática ortopédica na comunidade, fora dos grandes centros ${ }^{(10-12)}$.

Vitale et $\mathrm{al}^{(10)}$ afirmaram não haver correlação entre 0 número de especialistas em ombro e o volume de cirurgias de ombro em determinada área geográfica; além disso, que não é possível determinar a proporção de procedimentos cirúrgicos no ombro realizados por profissionais realmente capacitados para tais procedimentos. Lyman et $\mathrm{al}^{(11)}$.afirmaram que os melhores resultados das artroplastias de ombro estão associados ao maior volume cirúrgico Hammond et al $^{(12)}$ confirmaram esses dados e concluíram que cirurgiões que realizam muitas artroplastias de ombro anualmente apresentam melhores resultados e menor número de complicações quando em comparação com cirurgiões com baixo número anual de procedimentos

A partir dessas informações, idealizamos um projeto de desenvolvimento de nosso registro cirúrgico das artroplastias de ombro e, de forma contínua, documentando dados epidemiológicos a respeito de nossos pacientes e técnica cirúrgica empregada, a fim de possibilitar, futuramente, a avaliação dos resultados clínicos relacionados a esse procedimento. Autores já documentaram que registros cirúrgicos são o formato ideal para definir as características epidemiológicas de uma população específica submetida a determinado procedimento cirúrgico e, a partir daí, desenvolver avaliações funcionais relevantes, apontando os resultados, incidência de complicações e de revisões, objetivando identificar fatores preditivos de maus resultados e, dessa forma, permitir o aprimoramento da técnica cirúrgica e dos implantes ${ }^{(3,5,8)}$.
Apesar de a nossa casuística ser bastante relevante (145 pacientes operados em 18 meses), não possuímos meios para comparar nosso volume de indicações com a média nacional, uma vez que no Brasil não existe um registro nacional relacionado com o número de substituições articulares do ombro realizadas ao ano, além de o número de publicações a respeito de artroplastia do ombro em nosso país ser escasso. Numa revisão das publicações sobre o tema na Revista Brasileira de Ortopedia (RBO), somente seis artigos relacionados ao assunto foram encontrados $^{(13-18)}$. Ao compararmos nossos dados com séries de casos dos grandes centros internacionais de artroplastias, podemos afirmar que nossos números encontram-se dentro da média das substituições articulares de ombro publicadas na literatura especializada ${ }^{(3,8,19)}$. Porém, ao comparar a incidência de substituições artroplásticas do ombro com a de outras articulações, podemos afirmar que a incidência de artroplastias de ombro em todo mundo ainda é imensamente inferior à das artroplastias de joelho e quadril ${ }^{(20)}$. Essa diferença pode ser explicada pelo fato de a doença degenerativa do ombro ser mais bem tolerada pelo paciente, que adapta suas atividades da vida diária à limitação funcional do membro superior, e ainda por ser a doença muitas vezes menos frequente no ombro quando comparada com a do quadril e joelho. A artroplastia do ombro é um procedimento cirúrgico tecnicamente difícil quando comparado com o de outras artroplastias e, dessa forma, não sendo indicada aos pacientes com a frequência das outras substituições articulares ${ }^{(4)}$.

Em nossa casuística houve predomínio das hemiartroplastias (128 pacientes - 88\%) em relação às artroplastias totais (17 pacientes - $11,7 \%$ ) no contexto geral e em todos os grupos e subgrupos. Comparando nossos números com aqueles dos dois registros de artroplastia do ombro publicados até o momento na literatura ortopédica, identifica-se que nossos resultados estão de acordo com o registro escocês ${ }^{(11)}$ e são opostos aos dados citados por Santos et al ${ }^{(13)}$, referentes ao registro da Mayo Clinic. Existem poucos estudos randomizados comparando as artroplastias totais com as hemiartroplastias; todavia, atualmente há predileção pela artroplastia total nas principais séries sobre esse tema, que tem sido constantemente debatido na literatura especializada com argumentação bem estruturada de ambos os lados ${ }^{(21-22)}$. Esse fato, provavelmente, relaciona-se com a evolução técnica nas substituições glenoideas e na experiência dos cirurgiões, hoje em dia mais habilitados a realizar esse tipo de procedimento, o que estaria aumentando os índices de satisfação com a técnica empregada. Esse resultado contraria nossa casuística, na qual houve predomínio de hemiartroplastias, por nossas indicações respeitarem parâmetros 
bem restritos no que se refere à substituição da glenoide, uma vez que se trata de uma etapa técnica de alto grau de dificuldade, em que falhas podem acarretar um desastroso resultado funcional ao paciente. Os pacientes por nós operados apresentavam frequentemente lesões bastante evoluídas, com rupturas extensas do manguito rotador e, dessa forma, sem indicação para a substituição artroplástica da superfície da glenoide. Apesar disso, devemos admitir que, com nosso progresso técnico na realização desse procedimento, temos aumentado gradativamente o número de indicações de substituição da glenoide.

Embora tenhamos realizado poucas vezes a substituição do componente glenoideo, não subvalorizamos pequenas lesões articulares dessa superfície. Em nossa prática, temos utilizado técnica de microfratura nas lesões cartilaginosas pequenas. Nas extensas, em pacientes jovens, ora realizamos interposição biológica utilizando transplante de menisco lateral adquirido no Banco de Tecidos MúsculoEsqueléticos do INTO, ou fragmento de cápsula anterior obtida do próprio paciente durante o acesso deltopeitoral, separando-a do tendão do subescapular, ou apenas realizamos a técnica de reaming and run, procurando corrigir a versão e regularizar a superfície articular da glenoide, evitando a colocação do componente glenoideo com risco de soltura precoce em indivíduos jovens e ativos ${ }^{(23)}$.

Diversas séries de casos na literatura mundial têm documentado as doenças degenerativas como a principal indicação etiológica das artroplastias de ombro ${ }^{(3,8,19)}$. Adams et $\mathrm{al}^{(19)}$, em seu registro de artroplastia do ombro da Mayo Clinic, apresentaram como diagnóstico etiológico para indicar a artroplastia do ombro 57\% dos casos com diagnóstico de doença degenerativa primária, 13\% dos casos com artrite reumatoide, $25 \%$ com trauma agudo da extremidade proximal do úmero e 5\% com tumor músculo-esquelético. Sharmae e Dreghorn ${ }^{(8)}$, no registro escocês, descreveram a artrite reumatoide como diagnóstico mais frequente, seguido por trauma agudo, osteoartrose e osteonecrose. Em nossa casuística identificamos como diagnóstico mais frequente: as sequelas traumáticas (54 pacientes - 37\%), as lesões traumáticas agudas (fraturas) (48 pacientes - 33\%) e as doenças degenerativas (43 pacientes - 30\%). Esses dados provavelmente têm relação direta com as características assistenciais de nossa instituição, referência quaternária do Sistema Único de Saúde para procedimentos de alta complexidade em Ortopedia e Traumatologia. Essa diferença entre diagnósticos etiológicos nas indicações da substituição artroplástica do ombro possivelmente relaciona-se também com o perfil genético e os hábitos da população e com a área geográfica. Porém, fatores que justifiquem essa diferença ainda não foram confirmados na literatura.
Sem dúvida, a alta incidência de sequelas traumáticas da extremidade proximal do úmero em nossa casuística é um dado muito importante, não havendo dado comparativo na literatura ortopédica mundial com repercussão econômica e social em um país emergente como o Brasil. O grande número dessas lesões complexas é resultado de tratamentos, ora negligenciados, ora impróprios, para as lesões traumáticas agudas da extremidade proximal do úmero. Esse fato demonstra, em parte, a falência em que se encontra o sistema público de saúde em nosso país, principalmente em nosso Estado, onde a população de baixa renda não tem acesso a tratamento primário ortopédico de qualidade que permita recuperação funcional adequada e seu retorno a suas atividades laborativas prévias, criando um grande ônus econômico e social ao país. Em geral, são indivíduos economicamente ativos, com média de idade inferior à dos pacientes que comumente são submetidos a artroplastias; e, por não conseguirem atendimento ortopédico primário adequado para a lesão traumática primária, apresentam sequelas de alta complexidade, com alto grau de incapacidade funcional do membro superior. A única solução ortopédica é a realização da substituição articular do ombro e que, futuramente, poderá necessitar de revisões cirúrgicas em virtude do inevitável desgaste dos componentes protéticos. Acreditamos que investimentos no atendimento primário do trauma, tanto em recursos humanos, quanto tecnológicos, pode evitar essa tragédia social.

Nossa incidência de artroplastias, nas lesões traumáticas agudas da extremidade proximal do úmero, encontra-se dentro da média da literatura específica. Hoje, nesse contexto, as indicações das artroplastias de ombro são bem definidas e os resultados apresentam grande variação entre cirurgiões em virtude da alta complexidade técnica que a reconstrução do ombro representa, cursando com enorme número de variáveis, as quais são um desafio até para os cirurgiões mais experientes ${ }^{(24)}$.

Nossa incidência de pacientes com osteoartrose primária do ombro é discretamente inferior à da média mundial; acreditamos que esse fato ocorra em virtude de os pacientes apresentarem boa tolerância à limitação funcional dos membros superiores, em razão dos hábitos diários de nossa sociedade; por isso, muitos pacientes evitam um procedimento cirúrgico desse porte. Nossa casuística em pacientes com diagnóstico de artrite reumatoide contraria a tendência mundial ${ }^{(8)}$; talvez isso ocorra pelo baixo índice de encaminhamento de casos com esse diagnóstico a nosso hospital, ora pelo desconhecimento dessa ferramenta terapêutica pelo clínico assistente, ora porque o paciente reumático prefere ser submetido a substituições articulares dos membros inferiores, tolerando melhor as limitações degenerativas nos membros superiores. 
Outro dado relevante em nosso grupo de pacientes é a pequena incidência de artropatia do manguito rotador. A restrição em indicar a hemiartroplastia nesse grupo de pacientes baseia-se nos resultados funcionais insatisfatórios apresentados nas principais séries mundiais sobre esse tema, e devido ao surgimento de novos desenhos protéticos, como a cabeça tipo CTA ${ }^{\mathrm{R}(25)}$, e as próteses reversas ${ }^{(26)}$, que hoje são os implantes mais usados para substituição articular do ombro, com indicação precisa nesse grupo de doentes, proporcionando resultados funcionais quando comparados com os das hemiartroplastias convencionais.

Nos registros de artroplastias de ombro publicados na literatura ortopédica, a média de idade dos pacientes submetidos a esse procedimento foi de 65 anos no registro escocês ${ }^{(8)}$ e de 71 anos no registro da Clínica Mayo, segundo Santos et $\mathrm{al}^{(13)}$. Em nossa casuística, a média de idade dos 154 pacientes submetidos à artroplastia do ombro foi de 65 anos (30-84 anos). Ao analisarmos a média de idade por grupo, identificamos que a média de idade no grupo de sequelas traumáticas foi de 61 anos (30-81 anos), inferior à do grupo de pacientes com doença degenerativa, que foi de 64 anos (26-88 anos), e à do grupo de pacientes com trauma agudo, de 70 anos. Consideramos que esses dados corroborem a necessidade de tratamento primário adequado das lesões traumáticas da extremidade proximal do úmero, uma vez que essa lesão acomete pacientes jovens, prováveis candidatos, no futuro, à revisão cirúrgica. A artroplastia primária do ombro em pacientes com sequelas traumáticas é considerada um desafio técnico para a maioria dos cirurgiões experimentados; as revisões artroplásticas nesses pacientes serão um desafio ainda maior ${ }^{(7)}$.

Em nossa prática, num total de 154 artroplastias do ombro, 94 componentes umerais foram cimentados (61\%) e 51 componentes umerais não o foram (39\%). Na divisão por grupos, em 54 pacientes do grupo das sequelas traumáticas, 41 componentes umerais foram cimentados (76\%). No grupo das doenças degenerativas, em 44 pacientes apenas 12 componentes umerais foram cimentados (28\%). E no grupo de trauma agudo, em 48 pacientes, 41 componentes umerais foram cimentados (85\%). Nosso critério para a cimentação do componente foi subjetivo, baseado na qualidade óssea umeral. Sempre que o cirurgião julgou que o paciente apresentava o úmero com cortical fina e osso medular de má qualidade, ele optou pela cimentação do componente umeral. Outro critério utilizado foi em relação à idade do paciente, sendo evitado cimentar componentes umerais em jovens, devido à alta possibilidade de esses pacientes virem a ser submetidos a revisões cirúrgicas, onde a presença do componente cimentado dificultaria tecnicamente o procedimento, au- mentando sua morbidade. Wirth et al ${ }^{(27)}$ publicaram uma série de 58 artroplastias de ombro utilizando componentes umerais não cimentados associados a enxerto ósseo autólogo da cabeça umeral, não havendo afrouxamentos em um seguimento de cinco anos; concluíram que a não cimentação do componente umeral associada à enxertia óssea autóloga impactada é uma opção segura. Em nossa casuística, não utilizamos enxertia óssea impactada nos componentes umerais não cimentados, mas acreditamos ser essa mais uma ferramenta técnica disponível na substituição articular do ombro. Rahme et al ${ }^{(28)}$ compararam componentes umerais cimentados e não cimentados em pacientes com artrite reumatoide, que reconhecidamente apresentam má qualidade óssea, e encontraram resultados semelhantes quanto à incidência de afrouxamento. Acreditamos que, atualmente, na literatura mundial há predileção por componentes umerais não cimentados. Apesar de em nossa prática apresentarmos predileção por componentes cimentados, avaliaremos criticamente nossa indicação, a fim de modificarmos nossa conduta nas próximas séries.

Assim como outras substituições articulares, a artroplastia de ombro está associada a inúmeras complicações precoces e tardias, tais como: afrouxamento do componente glenoideo, instabilidade, fratura periprotética, lesão do manguito rotador, infecção, lesão neurovascular e disfunção do deltoide. Na literatura ortopédica, a incidência de complicações associadas à artroplastia de ombro tem alto índice de variação, de 0 a 62\%, com média de 14 a $16 \%$. De fato, a incidência exata das complicações relacionadas com as artroplastias de ombro não é conhecida devido ao fato de as séries de casos publicadas a respeito desse procedimento, em sua maioria, não apresentarem seguimento suficiente para essa afirmativa. Wirth et $\mathrm{al}^{(29)}$ afirmam que para se determinar a real incidência de complicações relacionadas à artroplastia do ombro, é necessário um seguimento mínimo de médio prazo (5-10 anos) ou longo prazo. Chin et $\mathrm{l}^{(30)}$ relataram incidência de complicações de 12\% em 431 artroplastias do ombro realizadas consecutivamente entre 1990 e 2000, com taxa de 3,9\% de revisões relacionadas com essas complicações. As complicações mais frequentes foram rupturas do manguito rotador, instabilidade e fraturas do úmero peroperatórias. Em nossa casuística, apresentamos no pós-operatório precoce complicações em cinco procedimentos: três relacionadas com o procedimento (uma lesão arterial e duas instabilidades) e duas complicações clínicas (uma infecção urinária e um óbito). Acreditamos que, futuramente, ao realizarmos a avaliação de nossos resultados, nossa incidência de complicações irá aumentar, em virtude das complicações tardias relacionadas com a falha mecânica e séptica do implante. 
A artroplastia de ombro é um procedimento tecnicamente exigente que está passando por grande evolução e inovação nos últimos anos, porém, apresenta documentação científica escassa na literatura especializada. Registros cirúrgicos são importantes na definição da epidemiologia das substituições articulares, além de provirem dados a respeito dos resultados e identificar fatores de risco de maus resultados.

Dessa forma, iniciamos nosso registro cirúrgico, a fim de documentar nossa experiência com artroplastia do ombro. A análise epidemiológica nos permitiu identificar as características dos pacientes, dos procedimentos cirúrgicos e das complicações imediatas das artroplastias do ombro realizadas em um hospital de alta complexidade.

Nossos resultados demonstram alta prevalência de pacientes jovens com sequelas traumáticas da extremidade proximal do úmero. Esse fato é preocupante, pois indica que estamos realizando substituições articulares do ombro em indivíduos mais jovens que o ideal e, portanto, futuramente, muitas artroplastias de revisão poderão ser necessárias, sendo essas cirurgias mais complexas e desafiadoras que as artroplastias primárias e com resultados menos satisfatórios. Além disso, destacamos a alta incidência de hemiartroplastias comparadas com artroplastias totais em nossa prática, fato oposto ao das grandes séries de artroplastia do ombro já publicadas.

Nosso registro de artroplastias do ombro é o passo inicial para que possamos realizar avaliações funcionais e documentar nossos resultados e, assim, determinar a eficácia desse procedimento, a incidência de complicações e necessidade de revisões.

\section{CONCLUSÃO}

As artroplastias de ombro tornaram-se um procedimento frequente na prática ortopédica. Registros cirúrgicos são importantes a fim de demonstrar essa evolução progressiva e permitir avaliações de resultados clínicos no futuro.

\section{REFERÊNCIAS}

1. Lugli T. Artificial shoulder joint by Pean (1893). The facts of an exceptional intervencion and the prosthetic method. Clin Orthop Relat Res. 1978;(133):215-8.

2. Neer CS 2 nd. Articular replacement of the humeral head. J Bone Joint Surg Am. 1955;37-A(2):215-28.

3. Jain NB, Higgins LD, Guller U, Pietrobon R, Katz JN. Trends in the epidemiology of total shoulder arthroplasty in the United States from 1990-2000. Arthritis Reum. 2006;55(4):591-7.

4. Matsen FA 3rd, Rockwood CA Jr, Wirth MA, Lippitt SB, Parson M. Glenohumeral arthritis and its management, In: Rockwood CA Jr, Matsen FA 3rd. The Shoulder. 3rd ed. Philadelphia: Sauders; 2004. p. 879-1008.

5. Maloney WJ. National joint replacement registries: Has the time come? J Bone Joint Surg Am. 2001;83(10):1582-5.

6. Neer CS 2nd. Displaced proximal humeral fractures. I. Classification and evaluation. J Bone Joint Surg Am. 1970;52(6):1077-89.

7. Boileau P, Trojani C, Walch G, Krishnam SG, Romeo A, Sinnerton R. Shoulder arthroplasty for the treatment of the sequelae of fractures of the proximal humerus. J Shoulder Elbow Surg. 2001;10(4):299-308.

8. Sharma S, Dreghorn CR. Registry of shoulder artroplasty - the Scottish experience. Ann R Coll Surg Engl. 2006;88(2):122-6.

9. Hasan SS, Leith J, Smith KL, Matsen FA 3rd. The distribution of shoulder replacement among surgeons and hospitals is significantly different than that of hips or knees replacements. J Shoulder Elbow Surg. 2003;12(2):164-9.

10. Vitale MG, Krant JJ, Gelijns AC, Heitjan DF, Arons RR, Bigliani LU, et al. Geographic variations in the rates of operative procedures involving the shoulder, including total shoulder replacement, humeral head replacement and rotator cuff repair. J Bone Joint Surg Am. 1999;81(6):761-72.

11. Lyman S, Jones EC, Bach PB, Peterson MGE, Marx RG. The association between hospital volume and total shoulder arthroplasty outcomes. Clin Orthop Relat Res 2005;(432):132-7.

12. Hammond JW, Queale WS, Kim TK, McFarland EG. Surgeon experience and clinical and economic outcomes for shoulder arthroplasty. J Bone Joint Surg Am. 2003;85(12):2318-24.

13. Santos PS, Bonomin C, Sobania LC, Otsuka N, Sobania RL, Ernlund LSR, et al. Hemiartroplastia em fratura e fratura-luxação do ombro. Rev Bras Ortop. 1994;29(9):651-5.

14. Rodrigues SA, Lemos JA, Reis ML. Hemiartroplastia do ombro não cimentada com prótese Global. Rev Bras Ortop. 1997;32(9):703-6.

15. Checchia SL, Doneaux P, Myasaki NA, Pombo EM, Meirelles MS Jr. Osteonecrose da cabeça do úmero: resultados das artrolastias. Rev Bras Ortop. 2001;36(1/2):29.

16. Veado MAC, Machado LP, Soares CG, Souza SV. Avaliação da função do ombro pós hemiartroplastia em fratura em 3 e 4 partes do úmero proximal. Rev Bras Ortop. 2001;36(5):161-6.
17. Checchia SL, Doneaux P, Miyasaki AN, Fregonese M, Silva LA, Faria FN, et al. Tratamento das fraturas do terço proximal do úmero com a prótese parcial Eccentra®. Rev Bras Ortop 2005;40(3):130-40.

18. Checchia SL, Doneaux P, Miyasaki AN, Fregonese M, Silva LA, Maschietto E, et al. Avaliação de resultados em artroplastia total do ombro. Rev Bras Ortop. 2006;41(5):173-80.

19. Adams JE, Sperling JW, Hoskin T, Melton $3^{\text {rd }}$ LJ, Cofield RH. Shoulder arthroplasty in Olmsted County, Minnesota, 1976-2000: a population-based study. J Shoulder Elbow Surg. 2006;15(1):50-5.

20. Soderman $\mathrm{P}$, Malchau $\mathrm{H}$, Heberts $\mathrm{P}$, Johnell O. Are the findings in the Swedish National total hip arthroplasty register valid? A comparison between the Swedish National total hip arthroplasty register, and the National Discharge register and the National Death register. J Arthoplasty. 2000;15(7):884-9.

21. Bryant D, Litchfield R, Sandow M, Gartsman GM, Guyatt G, Kirkley A. A comparison of pain, strength, range of motion and functional outcome after hemiarthroplasty and total shoulder arthroplasty in patients with osteoarthritis of the shoulder: a systematic review and meta-analysis. J Bone Joint Surg Am. 2005;87(9):1947-56.

22. Wirth MA, Tapscott RS, Southworth C, Rockwood CA Jr. Treatment of glenohumeral arthritis with a hemiarthroplasty: a minimum 5 year follow-up outcome study. J Bone Joint Am. 2006;88(5):964-73.

23. Cole BJ, Yankle A, Provencher MT: Nonarthroplasty alternatives for the treatment of glenohumeral arthritis. J Shoulder Elbow Surg. 2007;16(5 Suppl):231-40.

24. Loew M, Heit Kemper S, Parsch D, Schneider S, Richert M. Influence of the design of the prosthesis on the outcome after hemiarthroplasty of the shoulder in dispplaced fractures of the head of humerus. J Bone Joint Surg Br. 2006;88(3):345-50.

25. Visotsky JL, Basamania C, Seebauer L, Rockwood CA Jr, Jensen KL. Cuff tear arthropathy: pathogenesis, classification and algorithm of treatment. J Bone Joint Surg Am. 2004;86 Suppl 2:35-40.

26. Boileau P, Watkinson D, Hatzidaktis AM, Hovorka I. Neer Award 2005: the Grammont reverse shoulder prothesis: Results in cuff tear arthritis, fracture sequelae and revision arthroplasty. J Shoulder Elbow Surg. 2006; 15(5):527-40.

27. Wirth MA, Lim M, Southworth C, Loredo R, Kaar TK, Rockwood CA Jr. Compaction bone-grafting in prosthetic shoulder arthroplasty. J Bone Joint Surg Am. 2007;89(1):49-57.

28. Rahme H, Mattsson P, Wikblad B, Larsson S. Cemented and press-fit humeral stem fixation provides similar results in Rheumatoid patients. Clin Orthop Relat Res. 2006;(448):28-32.

29. Wirth MA, Rockwood CA Jr. Complications of total shoulder replacement arthroplasty. J Bone Joint Surg Am. 1996;78(4):603-16.

30. Chin PY, Sperling JW, Cofield RH, Schleck C. Complications of total shoulder arthroplasty: are they fewer or different? J Shoulder Elbow Surg. 2006;15(1):19-22. 